IN PRACTICE

\title{
Monitoring STI prevalence using telephone surveys and mailed urine specimens: a pilot test
}

\author{
E Eggleston, C F Turner, S M Rogers, A Roman, W C Miller, M A Villarroel, L Ganapathi
}

Sex Transm Infect 2005;81:236-238. doi: 10.1136/sti.2004.011080

Objectives: This pilot test assessed the feasibility of a cost effective population based approach to STI monitoring using automated telephone interviews, urine specimen collection kits sent out and returned by US Postal Service mail, and monetary incentives to motivate participation.

Methods: 100 residents of Baltimore, MD, USA, completed an automated telephone survey and agreed to mail in a urine specimen to be tested for chlamydia and gonorrhoea. Participants were paid $\$ 10$ for completing the survey and $\$ 40$ for mailing the specimen.

Results: $86 \%$ of survey participants mailed in a urine specimen for testing.

Conclusions: Automated telephone surveys linked with testing of mailed-in urine specimens may be a feasible lower cost (relative to household surveys) method of estimating infection prevalences in a population.

n 2001, over one million cases of Neisseria gonorrhoeae (GC) or Chlamydia trachomatis (CT) were reported to the Centers for Disease Control. ${ }^{1}$ Reported data are likely to under-represent actual infection rates in the United States by $50 \%$ or more. ${ }^{2} \mathrm{~A}$ substantial proportion of CT and GC infections remain unidentified and untreated because they are asymptomatic. Moreover, many diagnosed infections are not reported.

Population based surveys in which participants give urine specimens to be tested for sexually transmitted infections (STIs) provide an opportunity to measure the prevalence of both symptomatic and undiagnosed, asymptomatic infections. These surveys suggest the presence of a large hidden epidemic of asymptomatic and untreated infections. ${ }^{3}$ The high cost of household surveys, however, makes their routine use impractical.

A number of studies, in both the United States and Europe, have assessed the feasibility of STI screening through the testing of mailed urine specimens collected in the home. However, none of these studies was conducted among a population based sample, and many reported very low participation rates. ${ }^{4-9}$ None of these studies used financial incentives to motivate participation.

We report results of a pilot test of a cost effective population based approach to STI monitoring that incorporates automated telephone interviews, urine specimen collection kits sent out and returned by US Postal Service mail, and monetary incentives to motivate participation. The intent of this pilot test was to obtain an estimate of the proportion of telephone survey respondents who would agree to provide a urine specimen, follow the mail-in procedures, and have their specimen arrive intact at the testing laboratory.

\section{MATERIALS AND METHODS}

The pilot test was conducted between June and August 2003. The target population was Baltimore, MD, USA, in adults aged 18-35 years residing in telephone accessible households with touch-tone telephones (93\% of Baltimore households report being telephone accessible). ${ }^{10}$ The sample was restricted to landline telephone numbers for which linked residential addresses were commercially available. This restriction allowed us to geocode the residential addresses and then stratify the sample to obtain equal numbers of residences in high, medium, and low income (2000) US census tracts. Our first stage sample selection used a list assisted, probability sampling technique that included an initial pre-screening of sampled numbers to increase the probability of reaching households with 18-35 year olds. Our second stage selection used quota sampling to obtain roughly equivalent numbers of respondents by sex and the socioeconomic status of their census tract. Fifty one study participants were female and 49 were male; 36 were from the high SES stratum, 31 from the middle SES stratum, and 33 from the lowest SES stratum.

Telephone recruitment for this pilot was purposive and was conducted until 100 respondents were obtained. The intent of this pilot was not to learn what our survey response rate would be, but to determine the proportion of survey respondents who would provide a urine specimen. Our previous experience with the 1999-2000 National STD and Behavior Measurement Experiment ${ }^{11}{ }^{12}$ indicates that survey staff can achieve response rates in excess of $60 \%$ in a national, interviewer administered, random digit dial telephone survey, with no financial incentive offered for participation.

All sampled households were sent a lead letter that described the study and informed residents that a telephone interviewer would be calling to request their participation in a health behaviour study. Interviewers called sampled telephone numbers until the phone was answered, or until the number had been called a minimum of 10 times without being answered (letting the telephone ring approximately 10 times per call attempt). One person per household was interviewed. In sampled residences with more than one person aged 18-35 years, any eligible household member available at the time of the call was interviewed. A telephone respondent's age was requested before describing the study in detail and revealing the financial incentive. People who declined to participate were not re-contacted, and no callbacks were made to households in which an 18-35 year old was not present at the time of the call. The telephone interviewer requested the eligible person's cooperation in completing a telephone survey and mailing in a urine specimen to be tested for gonorrhoea and chlamydia. Participants were told that they would be reimbursed for their participation in the study. They were also informed that they would be re-contacted if their test result was positive and that, as required by law, names and contact information

Abbreviations: LCR, ligase chain reaction; STI, sexually transmitted infections 
of people who tested positive would be reported to the local health department.

The survey took 10-15 minutes to complete and collected information about participants' sexual behaviour, STI symptoms and history, and demographic characteristics. Interviewers obtained oral consent for the survey, and interviews were conducted by transferring respondents to RTI's T-ACASI (telephone audiocomputer assisted self interviewing) system. T-ACASI technology offers an interview mode that is considerably less expensive than in-person surveys, can be conducted more rapidly, does not require geographic clustering of samples (with a resultant loss in sample precision), affords participants privacy without requiring literacy, and streamlines data collection and analysis. ${ }^{11} 12$

Each participant was mailed a urine specimen collection kit specially designed for in-home use, a written consent form, urine collection instructions, and a $\$ 10$ money order for completing the telephone survey. Collection kits were mailed to participants a maximum of 4 days after the T-ACASI interview. Participants mailed urine specimens in preaddressed postage paid shipping cartons to the University of North Carolina-Chapel Hill (UNC) STD Clinical Research Center via US Postal Service (USPS) first class mail. The mailing followed USPS and Department of Transportation guidelines for mailing of diagnostic specimens. A preservative, DNA/RNA Protect, was included in the specimen cup to protect the urine specimen from degradation for 7-10 days without refrigeration. Urine specimens were tested for chlamydia and gonorrhoea using a ligase chain reaction (LCR) assay. Testing was not conducted on specimens submitted without a signed consent form. Participants received $\$ 40$ for mailing in the urine specimen. Participants who tested positive for CT or GC were personally contacted and referred for counselling and medical care to a Baltimore City Health Department clinic.

\section{RESULTS}

Eighty six per cent of the pilot survey participants mailed in a urine specimen for GC and CT testing. No specimens were lost during shipping, and all were adequate for testing. (Leakage occurred with two specimens, but testing was still possible.) Of the 14 participants who did not provide specimens, four explicitly declined and 10 simply did not mail in a specimen. Survey participants with more than a high school education were more likely than less educated participants to mail in urine specimens, $86 \% v 67 \%$. There were no statistically significant differences between those who mailed in urine specimens and those who did not in terms of sex, race, age group, or household income stratum. Two of 86 people, one male and one female, tested positive for chlamydia; none tested positive for gonorrhoea.

The majority of participants (59\%) mailed in a urine specimen without any reminders. Most of the re-contacted participants required only one or two calls. Eight participants were re-contacted three to five times before they mailed in their specimens. In three cases, respondents mailed in specimens after being mailed a second urine collection kit. Urine specimens were received at the UNC laboratory an average of 16 days (minimum 4 days, maximum 47 days) after the survey interview was completed.

\section{DISCUSSION}

We tested the feasibility of a research design that combines T-ACASI telephone surveys with mailed-in urine specimen collection in order to monitor the prevalence of two common STIs. Our findings suggest that a substantial majority (86\%) of respondents will provide urine specimens when an incentive is paid, reminder calls are made, and positive participants are informed of their test results. This compares favourably with the $80 \%$ in-person specimen collection rate obtained in the 1997-8 Baltimore STD and Behavior Study (BSBS). ${ }^{3}$ Moreover, this new data collection paradigm is less costly than a comparable in-person survey. A random digit dial telephone survey and specimen kit shipping cost approximately $\$ 250-\$ 300$ per person, while costs for a field survey are approximately $\$ 750-1000$ per person. (These estimates are approximate and are subject to variations in study design.)

Obviously, monitoring STI prevalence using specimens collected and mailed by participants themselves has some drawbacks. Because participants collect their own urine specimens, there is a lack of control over the collection process. It is possible that specimens submitted are not the respondents' specimens or the specimens are not urine. Future studies will test for this latter possibility. Further, for a minority of participants, several weeks elapsed between the date of the telephone survey and the date the urine specimen was provided. Among these participants, a detected infection may not have been present at the time of the survey, and survey responses thus may not reflect the participant's most relevant risk behaviour. However, the advantages of population based monitoring and the low cost, relative to household surveys, of telephone surveys with mailed urine specimens outweigh the disadvantages of this method of STI monitoring. Using automated telephone surveys linked with testing of mailed urine specimens is a feasible method of estimating infection prevalences and may be a practical component of an STI control programme. Because this pilot was not conducted among a random sample, the infection rate among study participants cannot be generalised to other populations or compared to the prevalence estimates obtained in the BSBS. A larger study, conducted on a random sample of the Baltimore population, will provide further information about the prevalence of these infections in the general population. ${ }^{13}$

\section{CONTRIBUTORS}

EE, CT, SR, WM, and AR conceptualised and designed the study and relevant analyses; EE, SR, and CT designed the questionnaire; AR oversaw sample specification and telephone recruiting and interviewing; MV and EE managed and analysed the data; EE was the primary writer of the paper; CT and SR contributed to revisions of the paper; WM, AR, and MV reviewed the paper; LG designed the T-ACASI program, managed T-ACASI data, and reviewed the paper.

\section{ACKNOWLEDGEMENTS}

The authors thank Phil Cooley, Alia Al-Tayyib, and Sheping Li for assistance with T-ACASI programming and data management; Vickie Stringfellow for management of telephone interviewing; Sarah Mobius for assistance with fieldwork logistics; and John Schmitz and Marcia Stedman for overseeing laboratory testing. We also thank Emily Erbelding, of the Baltimore City Health Department and Johns Hopkins University for coordinating medical care offered to study participants.

All study protocols and materials were approved by the institutional review boards of Research Triangle Institute, the University of Massachusetts-Boston and the University of North Carolina-Chapel Hill.

\section{Authors' affiliations}

E Eggleston, C F Turner, S M Rogers, M A Villarroel, Health and Behavior Measurement Program, Research Triangle Institute, Washington DC, USA

C F Turner, Queens College and the Graduate Center of the City University of New York, NY, USA

A Roman, Center for Survey Research, University of MassachusettsBoston, USA

W C Miller, Departments of Medicine and Epidemiology, University of North Carolina-Chapel Hill, USA 
L Ganapathi, Research Computing Division, Research Triangle Institute, Research Triangle Park, NC, USA

Correspondence to: Elizabeth Eggleston, Research Triangle Institute, 1615 M Street NW, Suite 740, Washington DC, 20036, USA; eeggleston@rti.org

Accepted for publication 21 August 2004

\section{REFERENCES}

1 Centers for Disease Control and Prevention. National Center for HIV, STD, and TB Prevention. Division of Sexually Transmitted Diseases. STD Surveillance 2001. Available at www.cdc.gov/std/stats/TOC2001.htm, accessed 29 August, 2003

2 Institute of Medicine, Committee on Prevention and Control of Sexually Transmitted Diseases. In: Eng TR, Butler WT, eds. The hidden epidemic: confronting sexually transmitted diseases. Washington, DC: National Academy Press, 1997:28-9, 70.

3 Turner CF, Rogers SM, Miller HG, et al. Untreated gonococcal and chlamydial infection in a probability sample of adults. JAMA 2002;287:726-33.

4 Bloomfield PJ, Kent C, Campbell D, et al. Community-based chlamydia and gonorrhea screening through the United States mail, San Francisco. Sex Transm Dis 2002;29:294-7.

5 Bloomfield PJ, Steiner KC, Kent CK, et al. Repeat chlamydia screening by mail, San Francisco. Sex Transm Infect 2003;79:28-30.
6 Rogstad KE, Bates SM, Partridge S, et al. The prevalence of chlamydia trachomatis infection in the male undergraduates: a postal survey. Sex Transm Infect $2001 ; 77: 111-13$.

7 MacLeod J, Rowsell R, Horner $\mathrm{P}$, et al. Postal urine specimens: are they a feasible method for genital chlamydia information screening? Br J Genl Pract 1999;49:455-8.

8 Stephenson J, Carder C, Copas A, et al. Home screening for chlamydial genital infection: is it acceptable to young men and women? Sex Transm Infect 2000;76:25-7.

9 Østergaard L, Moller JK, Andersen B, et al. Diagnosis of urogenital Chlamydia trachomatis infection in women based on mailed samples obtained at home: multipractice comparative study. BMJ 1996:313:1186-9.

10 US Bureau of the Census, State and County Quick Facts. 2003. Available at quickfacts.census.gov/qfd/states/24/24510. html, accessed 1 August, 2003.

11 Villarroel MA, Turner CF, Eggleston E, et al. Same-gender sex in the USA: impact of interview methodology and time on prevalence estimates. Presented at the American Public Health Association 130th Annual Meeting, 2002, November 9-13, Philadelphia, PA (abstract \#44782).

12 Al-Tayyib AA, Turner CF, Rogers SM, et al. Impact of T-ACASI on the measurement of sexual practices. Presented at the American Public Health Association 130th Annual Meeting, 2002, November 9-13, Philadelphia, PA (abstract \#44624).

13 Eggleston E, Turner CF, Rogers SM, et al. Monitoring STls in the population. Grant application submitted to the National Institutes of Health, 1 September 2004. Study period to begin April 2005. 